\title{
Symmetry Breaking in Wrinkling Patterns: Gyri Are Universally Thicker than Sulci
}

\author{
Maria Holland, ${ }^{1,4}$ Silvia Budday, ${ }^{2}$ Alain Goriely, ${ }^{3}$ and Ellen Kuhl ${ }^{1}$ \\ ${ }^{1}$ Mechanical Engineering, Stanford University, Stanford, California 94305, USA \\ ${ }^{2}$ Mechanical Engineering, FAU Erlangen, 91058 Erlangen, Germany \\ ${ }^{3}$ Mathematical Institute, University of Oxford, Oxford OX2 6GG, United Kingdom \\ ${ }^{4}$ Aerospace and Mechanical Engineering, University of Notre Dame, Indiana 46556, USA
}

(Received 6 March 2018; revised manuscript received 20 September 2018; published 28 November 2018)

\begin{abstract}
Wrinkling instabilities appear in soft materials when a flat elastic layer on an elastic substrate is sufficiently stressed that it buckles with a wavy pattern to minimize the energy of the system. This instability is known to play an important role in engineering, but it also appears in many biological systems. In these systems, the stresses responsible for the wrinkling instability are often created through differential growth of the two layers. Beyond the instability, the upper and lower sides of the elastic layer are subject to different forces. This difference in forces leads to an interesting symmetry breaking whereby the thickness becomes larger at ridges than at valleys. Here we carry out an extensive analysis of this phenomenon by combining analytical, computational, and simple polymer experiments to show that symmetry breaking is a generic property of such systems. We apply our idea to the cortical folding of the brain for which it has been known for over a century that there is a thickness difference between gyri and sulci. An extensive analysis of hundreds of human brains reveals a systematic region-dependent thickness variation. Our results suggest that the evolving thickness patterns during brain development, similar to our polymer experiments, follow simple physics-based laws: Gyri are universally thicker than sulci.
\end{abstract}

DOI: 10.1103/PhysRevLett.121.228002

Introduction.-The wrinkling of a stiff elastic layer on an soft elastic substrate is a universal pattern-forming instability that has been extensively studied in physical, engineering, and biological systems [1-3]. Wrinkling is particularly relevant in biology, where a central question is to understand the interplay of genetic, biochemical, geometric, and physical factors during morphogenesis. Bilayer instabilities in these systems can be created by differential growth between layers and, depending on material and geometric parameters, display a wide variety of instabilities $[4,5]$ that have important applications in plants [6], seashells [7], and embryogenesis [8], where small variations in thickness can have large effects on the evolution of shape.

In the particular case of brain morphogenesis, it was proposed by the Swiss anatomist Wilhelm His in his 1874 essay "Unsere Körperform" that Entwicklungsmechanik, developmental mechanics, is the key driver for the characteristic folding pattern of our brain [9]. Motivated by this idea [10], there is now a common understanding [11-15] that the folding pattern of our brain is the result of differential growth and morphoelastic instabilities [16-18].

Published by the American Physical Society under the terms of the Creative Commons Attribution 4.0 International license. Further distribution of this work must maintain attribution to the author(s) and the published article's title, journal citation, and DOI.
More recently, this notion has been supported by wrinkling in brain organoids [19] and by a large-scale transcriptomic analysis that found discrete domains of gene expression in the developing gyrencephalic cortex of ferrets but not in the lissencephalic cortex of mice [20].

The phenomenon of cortical folding is therefore an excellent test bed to study the role of physics in morphogenesis. For instance, both geometry and mechanics are important to explain that dolichocephalic elongated brains tend to be more longitudinally folded than brachycephalic round brains [21]. We also know that small perturbations during cortical development can produce significant alterations in cortical thickness, which are closely associated with neurodevelopment disorders like lissencephaly and polymicrogyria but also with autism spectrum disorders [22] and schizophrenia [23].

In the living brain, the cortical thickness is the distance between the white matter and pial interface [24]. It changes only marginally with brain size and varies between 1.5 and $4.5 \mathrm{~mm}$ in the human brain [25]. An interesting observation is that gyri, the elevated visible part of the wavy pattern, are usually thicker than sulci, the lower hidden part of the pattern. This difference was first noted more than a century ago by Brodman [26] and confirmed more recently by various authors [14,24,27].

Motivated by these observations, our first problem is to explore whether a physical bilayer system also exhibits the same symmetry-breaking mechanism: Do wrinkling 
patterns have a natural and generic asymmetry between maxima and minima? A second problem is to determine whether the difference in thickness reported a century ago is indeed present in human brains and whether it can be explained, at least in part, by physical arguments. To answer these questions, we study the problem from four different perspectives: First, we theoretically analyze the bifurcation of a bilayer system with differential growth. Second, we simulate a similar system and explore the same solution in a computational setting. Third, we perform polymer experiments that generate a similar wrinkling instability. Fourth, we return to the cortex and perform a systematic analysis of the gyri and sulci of human brains.

Theoretical model.-We consider an idealized but generic analytical version of the problem. We model the symmetry breaking during folding as a morphoelastic instability caused by the homogeneous growth of an elastic layer on an elastic substrate modeled as an elastic half space in the plane. We use the theory of morphoelasticity [28-30] and decompose the deformation gradient $\mathbf{F}=\operatorname{Grad}(\boldsymbol{\chi})$ of the deformation $\mathbf{x}=\boldsymbol{\chi}(\mathbf{X})$ into an elastic contribution $\mathbf{A}$ and a growth contribution $\mathbf{G}$ so that $\mathbf{F}=\mathbf{A} \cdot \mathbf{G}$. Constitutively, only the elastic contribution $\mathbf{A}$ generates stresses. We assume a neo-Hookean incompressible material behavior, $\mathbf{T}=\mu \mathbf{A}^{T} \cdot \mathbf{A}-p \mathbf{I}$, where $\mu$ is the shear modulus, $\mathbf{I}$ is the second-order unit tensor, and $p$ is the Lagrange multiplier associated with the incompressibility constraint. The stresses enter the mechanical equilibrium equation as $\operatorname{div} \mathbf{T}=\mathbf{0}$.

The key parameter of the model is the stiffness ratio $\beta=\mu_{f} / \mu_{s}$ between the layer and substrate. We assume that the layer grows isotropically and morphogenetically as $\mathbf{G}=\vartheta \mathbf{I}$, where $\vartheta$ is the growth multiplier. We postulate that the layer grows at a constant rate $\dot{\vartheta}$ and that the substrate is purely elastic and does not grow, $\vartheta=1$. To compute the critical growth value $\vartheta_{c}$ at which the wrinkling instability sets in, we adopt a variational method that probes the stability of the homogeneous solution by studying emerging folding modes with the lowest elastic energy [31]. This method given in Supplemental Material [32] also provides a description of the shape of the folds and the deformations of each layer for values of the growth factor $\vartheta$ larger than, but close to, $\vartheta_{c}$. The analytical solution provides the thicknesses of the gyri and sulci as a function of the amplitude up to quadratic order: Initially, before growth, the layer is in the domain $(Y, X) \in[0,1] \times \mathbb{R}$ and the substrate in the domain $(Y, X) \in(-\infty, 0] \times \mathbb{R}$. To first order, the shape of the wrinkling mode is written in mixed coordinates $(x, Y)$ as $y(x, Y)=\vartheta^{2} Y+A k F_{1}(Y) \cos (k x)$, where $A$ is the amplitude of the wrinkle, $F_{1}(Y)$ describes variations in the vertical direction, $Y$ is the height of a material point in the initial configuration, and $\vartheta=\vartheta_{c}$ in the layer and $\vartheta=1$ in the substrate. The thicknesses of the gyri and sulci, normalized by the thickness at the onset of wrinkling, are defined, respectively, by

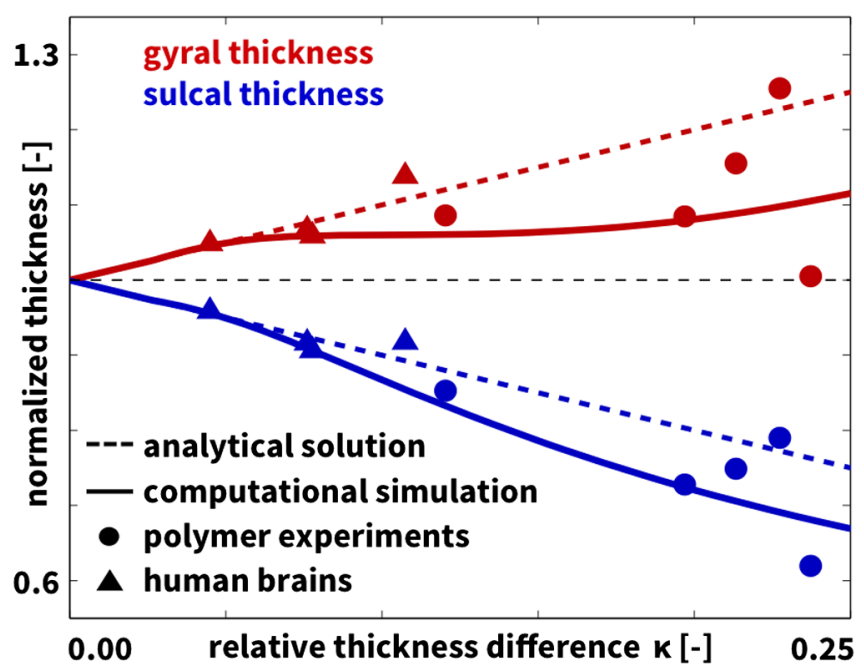

FIG. 1. Symmetry breaking in wrinkling patterns. Gyri are thicker than sulci in our analytical solution, computational simulation, polymer experiments, and human brain analysis. All gyral and sulcal thicknesses are normalized by the thickness at the onset of the instability and are shown as functions of the relative thickness difference.

$t_{g}=\frac{y\left(\pi / k_{c}, 1\right)-y\left(\pi / k_{c}, 0\right)}{\vartheta_{c}^{2}}, \quad t_{s}=\frac{y(0,1)-y(0,0)}{\vartheta_{c}^{2}}$.

In the linear approximation, the thicknesses are given by

$$
t_{g}=1+a A, \quad t_{s}=1-a A .
$$

The coefficient $a>0$ depends on the stiffness ratio $\beta$ and is positive for all stiffness ratios for which a wrinkling instability takes place, in this setting for $\beta \gtrsim 0.544$ (the Biot limit [33] explained in Supplemental Material [32]). Furthermore, for $\beta \gtrsim 0.943$, the wrinkling instability is a supercritical pitchfork bifurcation [5,34], and the coefficient $a$ decreases as $\beta$ increases. Hence, we expect the wrinkling pattern to be stable at onset, and we conclude that, close to the bifurcation, gyri will increase in thickness while sulci decrease. We introduce the relative thickness difference

$$
\kappa=\frac{t_{g}-t_{s}}{t_{g}+t_{s}}=a A,
$$

such that the gyral and sulcal thicknesses $t_{g}=1+\kappa$ and $t_{s}=1-\kappa$ depend only on geometric quantities. Figure 1 illustrates our analytical thickness estimates as dashed red and blue lines. We will now compare them against our computational simulations, polymer experiments, and human brain measurements.

Computational model.-To characterize gyral and sulcal thicknesses beyond the bifurcation point, we simulate the growth of a stiff thin layer on top of a soft thick substrate, similar to the previous section, but now assuming slight compressibility for computational stability. We assume 


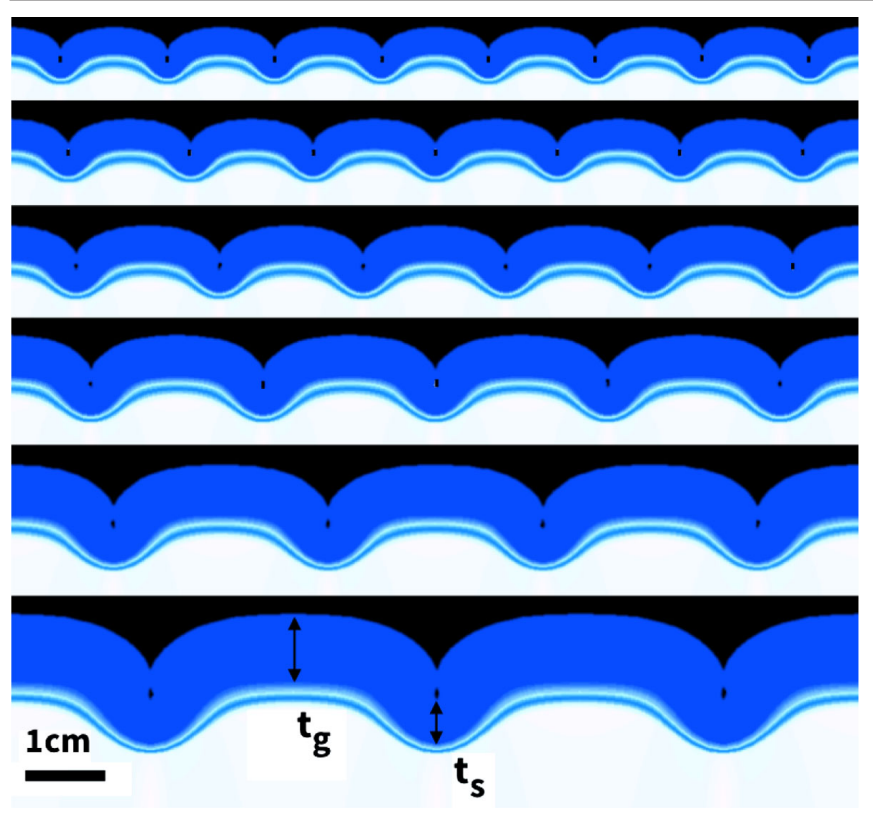

FIG. 2. Computational simulations. Folding patterns for varying initial layer thicknesses of 1.25, 1.43, 1.67, 2.00, 2.50, and $3.33 \mathrm{~mm}$ (from top to bottom) confirm that the wavelength scales linearly with the layer thickness and reveal that the gyral thickness $t_{g}$ is always larger than the sulcal thickness $t_{s}$.

a neo-Hookean compressible material behavior: $\mathbf{T}=$ $\mu \mathbf{A}^{T} \cdot \mathbf{A}+\left[\Lambda \ln \left(J^{e}\right)-\mu\right] \mathbf{I}$, where $\Lambda$ and $\mu$ are the elastic Lamé constants and $J^{e}=\operatorname{det}(\mathbf{A})$ is the Jacobian of the elastic tensor A. We create a finite element model of a bilayer system with varying layer thicknesses of $1.25,1.43$, $1.67,2.00,2.50$, and $3.33 \mathrm{~mm}$, discretize it with 5760 elements and 12050 degrees of freedom, and solve it using a custom-designed nonlinear finite element program [35]. We assume a plane strain state and fix all boundary nodes orthogonal to the boundary but allow them to slide freely along the edge. Motivated by the stiffness ratios in human brain tissue [36], we assume that the layer with $\mu=$ $0.30 \mathrm{kPa}$ and $\Lambda=2.79 \mathrm{kPa}$ is 3 times stiffer than the substrate with $\mu=0.10 \mathrm{kPa}$ and $\Lambda=0.93 \mathrm{kPa}$.

Figure 2 shows the results of our computational simulations at the first point of self-contact. Our simulations confirm the general notion that the wavelength scales linearly with the layer thickness. For increasing initial layer thicknesses [37], from 1.25, 1.43, 1.67, 2.00, and 2.50 to $3.33 \mathrm{~mm}$, the wavelength increases from 12.0, 13.7, $16.0,19.2$, and 24.0 to $32.0 \mathrm{~mm}$ at a constant thickness-towavelength ratio of 0.104 . For all six simulations with varying initial layer thicknesses, the gyral thickness $t_{g}$ was always larger than the sulcal thickness $t_{s}$. For the smallest layer thickness of $1.25 \mathrm{~mm}$, we record the gyral and sulcal thicknesses $t_{g}$ and $t_{s}$, normalize them by the layer thickness at the onset of wrinkling, and plot them versus the relative thickness difference $\kappa$ as solid curves in Fig. 1. The solid curves of our computational simulation show excellent

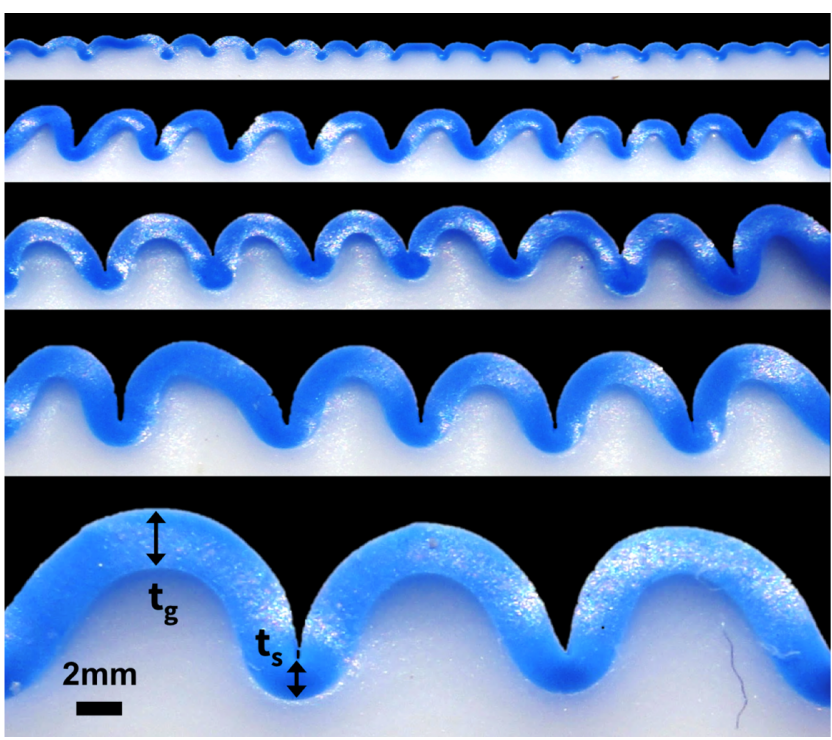

FIG. 3. Polymer experiments. Folding patterns for varying initial layer thicknesses of $0.3,0.6,0.8,1.0$, and $2.0 \mathrm{~mm}$ (from top to bottom) confirm that the wavelength scales linearly with the layer thickness and reveal that the gyral thickness $t_{g}$ is always larger than the sulcal thickness $t_{s}$.

agreement with the dashed lines of our analytical solution and reflect the nonlinear nature of the problem further away from the initial bifurcation point, here at relative thickness differences of $\kappa>0.05$.

Polymer experiments. - To experimentally characterize the effect of symmetry breaking, we create a bilayer system of room-temperature-vulcanization silicone rubber with a stiff thin layer on top of a prestretched soft thick substrate [38]. To cast the soft substrate, we mix a platinumcatalyzed silicone, Smooth-On Ecoflex 00-30, dye the mixture in white, pour it into a rectangular casting mold, and cure it for $5 \mathrm{~h}$ at room temperature. After curing, we prestretch the substrate uniaxially from its initial length $L$ to a prestretched length $l$ using a custom-built stretching device. To prepare the stiff layer, we mix a platinum silicone, Smooth-On Mold Star 20 T, dye the mixture in blue, pour it onto the stretched substrate, and cure it for $2 \mathrm{~h}$ at room temperature. To induce folding, we gradually release the prestretch. We take images of the folded bilayer and characterize the gyral and sulcal thicknesses using digital imaging techniques. We apply a constant prestretch of $\lambda=l / L=2.0$. At this prestretch level, the layer and substrate have stiffnesses of 324 and $69 \mathrm{kPa}$, respectively, which generates a stiffness contrast of 4.7, about twice the value as in the adult human brain [36].

Figure 3 and Table I summarize the results of our polymer experiments after releasing the prestretch. Our experiments confirm that the wavelength scales linearly with the layer thickness [37]. For all five experiments, the gyral thickness $t_{g}$ was always larger than the sulcal thickness $t_{s}$. For the five initial layer thicknesses, from $0.3,0.6,0.8$, and 1.0 to $2.0 \mathrm{~mm}$, 
TABLE I. Polymer experiments. Gyral and sulcal thicknesses and thickness ratios for varying initial layer thicknesses at a constant prestretch of $\lambda=2.0$ p; see Fig. 3 .

\begin{tabular}{lccc}
\hline \hline $\begin{array}{c}\text { Layer } \\
(\mathrm{mm})\end{array}$ & $\begin{array}{c}\text { Gyral thickness } \\
(\mathrm{mm})\end{array}$ & $\begin{array}{c}\text { Sulcal thickness } \\
(\mathrm{mm})\end{array}$ & Ratio \\
\hline 0.300 & $0.391 \pm 0.066$ & $0.307 \pm 0.058$ & 1.274 \\
0.600 & $0.781 \pm 0.062$ & $0.524 \pm 0.074$ & 1.490 \\
0.800 & $1.109 \pm 0.131$ & $0.719 \pm 0.124$ & 1.543 \\
1.000 & $1.506 \pm 0.063$ & $0.948 \pm 0.094$ & 1.589 \\
2.000 & $2.412 \pm 0.300$ & $1.487 \pm 0.147$ & 1.622 \\
\hline \hline
\end{tabular}

we record the gyral and sulcal thicknesses $t_{g}$ and $t_{s}$ in Table I, normalize them by the layer thickness at the onset of wrinkling that we infer from kinematic estimates, and plot them versus the relative thickness difference $\kappa$ as solid circles in Fig. 1. We conclude that our polymer experiments display a good agreement with the dashed and solid lines of our analytical solution and our computational model.

Human brains. - We now return to the initial problem of the human brain. To study the thickness asymmetry in the human cortex, we analyze magnetic resonance images of 564 healthy adult human brains from a public database [39]. We perform volumetric image segmentation and
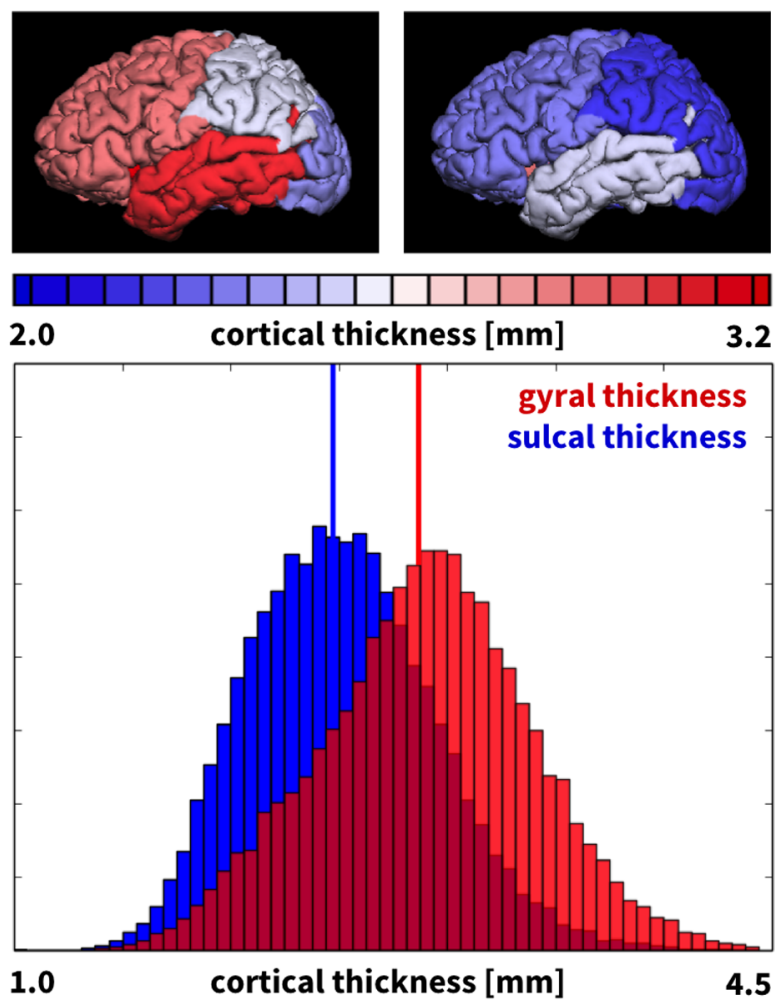

FIG. 4. Human brains. Mean gyral (top left) and sulcal (top right) thicknesses of the frontal, temporal, parietal, and occipital lobes. Histogram of gyral and sulcal thicknesses of 58 gyral regions and 62 sulcal regions for 564 healthy adult human brains (bottom). Vertical lines indicate the mean gyral and sulcal thicknesses of 2.87 and $2.47 \mathrm{~mm}$, respectively.
TABLE II. Human brains. Gyral and sulcal thicknesses and thickness ratios in the frontal, parietal, occipital, and temporal lobes, averaged over 564 human brains; see Fig. 4.

\begin{tabular}{lccc}
\hline \hline Lobe & $\begin{array}{c}\text { Gyral thickness } \\
(\mathrm{mm})\end{array}$ & $\begin{array}{c}\text { Sulcal thickness } \\
(\mathrm{mm})\end{array}$ & Ratio \\
\hline Frontal & $2.988 \pm 0.235$ & $2.410 \pm 0.217$ & 1.240 \\
Temporal & $3.118 \pm 0.300$ & $2.674 \pm 0.267$ & 1.166 \\
Parietal & $2.761 \pm 0.222$ & $2.365 \pm 0.229$ & 1.167 \\
Occipital & $2.557 \pm 0.218$ & $2.342 \pm 0.215$ & 1.092 \\
\hline \hline
\end{tabular}

cortical reconstruction using FREESURFER [40]. We adopt the Destrieux atlas [41] to parcellate the cortical surface into four lobes (frontal, temporal, parietal, and occipital) and 58 gyral and 62 sulcal regions. We determine the cortical thickness in each of these 67680 regions [24] and report the gyral and sulcal thicknesses both individually for each lobe and collectively in a histogram.

Figure 4 and Table II summarize the results of our human brain analysis. The histogram in Fig. 4 (bottom) shows that the mean thickness of all 32712 gyral regions of $2.87 \mathrm{~mm}$ was significantly larger, with a $p$ value of $p \ll 10^{-10}$, than the mean thickness of all 34968 sulcal regions of $2.47 \mathrm{~mm}$, with an overall gyral-to-sulcal thickness ratio of 1.162. The thickest region was region 18 of the Destrieux atlas [41], the short insular gyrus, with $3.75 \pm 0.71 \mathrm{~mm}$, and the thinnest region was region 57, the middle occipital sulcus and lunatus sulcus, with $1.89 \pm 0.42 \mathrm{~mm}$. The color-coded brains in Fig. 4 (top) highlight the mean gyral (left) and sulcal (right) thicknesses in the frontal, temporal, parietal, and occipital lobes. As Table II confirms, in each lobe, the mean gyral thickness was significantly larger $\left(p \ll 10^{-10}\right)$ than the mean sulcal thickness. The thickness ratio between gyri and sulci displayed an anterior-to-posterior gradient with 1.240 in the frontal lobe, 1.166 and 1.167 in the temporal and parietal lobes, and 1.082 in the occipital lobe. For all four lobes, we normalized gyral and sulcal thicknesses $t_{g}$ and $t_{s}$ from Table II and plotted them versus the relative thickness difference $\kappa$ as solid triangles in Fig. 1. We conclude that our human brain analysis displays an excellent agreement with the dashed and solid lines of our analytical solution and our computational model.

Discussion.-We studied the symmetry breaking during folding, analytically, computationally, experimentally, and anatomically. We observed, in Figs. 2-4, that the homogeneous system undergoes a unique symmetry breaking into a spatially heterogeneous system with discrete gyri and sulci. Strikingly, in all systems, the gyral thicknesses increases while the sulcal thicknesses decrease. This symmetry breaking emerges naturally, as we show in Fig. 1, even in the complete absence of local perturbations or nonuniform growth.

Our theoretical analysis indicates that our observed thickness variations decrease with an increasing stiffness ratio (see Supplemental Material [32]). This suggests that 
gyral-to-sulcal thickness variations are a low-stiffness-ratio phenomenon that is less pronounced, or even absent, in large-stiffness-ratio systems. We conclude that symmetry breaking is most visible in low-stiffness-ratio systems like the human brain.

We showed that the thickness ratio between gyri and sulci evolves dynamically and increases continuously with increasing growth. Figure 1 highlights this effect for both the dashed lines of the analytical solution and the solid lines of the computational simulation. This could explain why, in Table II, the gyral-to-sulcal thickness ratio is higher in the frontal lobe with a lower average cortical thickness than in the temporal lobe.

Conclusion.-A key question in brain morphogenesis is whether regional thickness variations between gyri and sulci are exclusively a result of genetic organization patterns. While we do not deny the importance of genetic effects during cortical folding, our study demonstrates that geometric and physical factors alone could induce cortical patterning and modulate cortical thickness. Our results suggest that genetic, geometric, and physical factors during brain development are closely interrelated. Our observations agree well with the mechanical feedback hypothesis in other model systems and could provide answers to the fundamental question in development of how an organ system knows where and when to grow. Beyond these implications in developmental biology, our study suggests that symmetry breaking in a wrinkling bilayer system is a universal phenomenon in which gyri generally tend to thicken while sulci tend to thin.

This work was supported by the NSF Graduate Research Fellowship and the Stanford Graduate and DARE Fellowships (M. H.), the Stanford Bio-X IIP and NSF Grant No. CMMI 1727268 (E. K.), and the EPSRC Grant No. EP/R020205/1 (A. G.).

[1] M. W. Moon, Sh. Lee, J. Y. Sun, K. H. Oh, A. Vaziri, and J. W. Hutchinson, Proc. Natl. Acad. Sci. U.S.A. 104, 1130 (2007).

[2] B. J. Kim, P. Kim, N. C. Pegard, S. J. Oh, C. R. Kagan, J. W. Fleischer, H. A. Stone, and Y. L. Loo, Nat. Mater. 10, 952 (2011).

[3] B. Li, Y. P. Cao, X. Q. Feng, and H. Gao, Soft Matter 8, 5728 (2012).

[4] M. A. Holland, B. Li, X. Q. Feng, and E. Kuhl, J. Mech. Phys. Solids 98, 350 (2017).

[5] A. Auguste, L. Jin, Z. Suo, and R. C. Hayward, Extreme Mech. Lett. 11, 30 (2017).

[6] M. A. Holland, T. Kosmata, A. Goriely, and E. Kuhl, Math. Mech. Solids 18, 561 (2013).

[7] R. Chirat, D. E. Moulton, and A. Goriely, Proc. Natl. Acad. Sci. U.S.A. 110, 6015 (2013).

[8] M. Ben Amar and F. Jia, Proc. Natl. Acad. Sci. U.S.A. 110, 10525 (2013).
[9] W. His, Unsere Körperform und das physiologische Problem ihrer Entstehung (F.C.W. Vogel, Leipzig, 1874).

[10] D. P. Richman, R. M. Stewart, J. W. Hutchinson, and V. S. Caviness, Science 189, 18 (1975).

[11] R. Toro and Y. Burnod, Cereb. Cortex 15, 1900 (2005).

[12] P. V. Bayly, R. Okamoto, G. Xu, Y. Shi, and L. A. Taber, Phys. Biol. 10, 016005 (2013).

[13] T. Sun and R. F. Hevner, Nat. Rev. Neurosci. 15, 217 (2014).

[14] T. Tallinen, J. Y. Chung, J. Biggins, and L. Mahadevan, Proc. Natl. Acad. Sci. U.S.A. 111, 12667 (2014).

[15] Y. Wang, J. Necus, M. Kaiser, and B. Mota, Proc. Natl. Acad. Sci. U.S.A. 113, 12820 (2016).

[16] A. Goriely, M. G. D. Geers, G. A. Holzapfel, J. Jayamohan, A. Jérusalem, S. Sivaloganathan, W. Squier, J. A. W. van Dommelen, S. Waters, and E. Kuhl, Biomech. Model. Mechanobiol. 14, 931 (2015).

[17] T. Tallinen, J. Y. Chung, F. Rousseau, N. Girard, J. Lefevre, and L. Mahadevan, Nat. Phys. 12, 588 (2016).

[18] E. Kuhl, Nat. Phys. 12, 533 (2016).

[19] E. Karzbrun, A. Kshirsagar, S. R. Cohen, J. H. Hanna, and O. Reiner, Nat. Phys. 14, 515 (2018).

[20] C. de Juan Romero, C. Bruder, U. Tomasello, J. M. SanzAnguela, and V. Borrell, EMBO J. 34, 1859 (2015).

[21] S. Budday, P. Steinmann, A. Goriely, and E. Kuhl, Extreme Mech. Lett. 4, 193 (2015).

[22] A. Y. Hardan, R. J. Jou, M. S. Keshavan, R. Varma, and N. J. Minshew, Psychiatr. Res. Neuroimag. 131, 263 (2004).

[23] R. J. Jou, A. Y. Hardan, and M. S. Keshavan, Schizophrenia research 75, 309 (2005).

[24] B. Fischl and A. M. Dale, Proc. Natl. Acad. Sci. U.S.A. 97, 11050 (2000).

[25] W. Welker, A Review of Determinants of Gyri and Sulci, in Cerebral Cortex, edited by E. G. Jones and A. Peters (Springer, New York, 1990), Vol. 8B.

[26] K. Brodmann, Vergleichende Lokalisationslehre der Großhirnrinde (Johann Ambrosius Barth Verlag, Leipzig, 1909).

[27] M. J. Razavi, T. Zhang, T. Liu, and X. Wang, Sci. Rep. 5, 14477 (2015).

[28] A. Goriely and M. Ben Amar, Phys. Rev. Lett. 94, 198103 (2005).

[29] S. Budday, C. Raybaud, and E. Kuhl, Sci. Rep. 4, 5644 (2014).

[30] A. Goriely, The Mathematics and Mechanics of Biological Growth, Springer Interdisciplinary and Applied Mathematics (Springer, New York, 2017).

[31] M. BenAmar and A. Bordner, J. Elast. 129, 213 (2017).

[32] See Supplemental Material at http://link.aps.org/ supplemental/10.1103/PhysRevLett.121.228002 for details on theoretical model, variational formulation, stability analysis, and layer stiffness.

[33] M. A. Biot, Appl. Sci. Res. 12, 168 (1963).

[34] J. W. Hutchinson, Phil. Trans. R. Soc. A 371, 20120422 (2013).

[35] S. Budday, P. Steinmann, and E. Kuhl, J. Mech. Phys. Solids 72, 75 (2014).

[36] S. Budday, G. Sommer, C. Birkl, C. Langkammer, J. Hayback, J. Kohnert, M. Bauer, F. Paulsen, P. Steinmann, E. Kuhl, and G. A. Holzapfel, Acta Biomater. 48, 319 (2017). 
[37] H. G. Allen, Analysis and Design of Structural Sandwich Panels (Pergamon Press, Oxford, 1969).

[38] S. Budday, S. Andres, B. Walter, P. Steinmann, and E. Kuhl, Phil. Trans. R. Soc. A 375, 20160163 (2017).

[39] C. Craddock, Y. Benhajali, C. Chu, F. Chouinard, A. Evans, A. Jakab, B.S. Khundrakpam, J. D. Lewis,
Q. Li, M. Milham, C. Yan, and P. Bellec, Front. Neuroinf. (2013).

[40] A. M. Dale, B. Fischl, and M. I. Sereno, NeuroImage 9, 179 (1999).

[41] C. Destrieux, B. Fischl, A. Dale, and E. Halgren, NeuroImage 53, 1 (2010). 